Micha F. Dorsch · Jenny A. Barrett

Richard A. Lawrance · Azhar Maqbool

Nigel P. Durham · Stacey Ellis · Nilesh J. Samani

Tim Bishop · Stephen G. Ball · Anthony J. Balmforth

Alistair S. Hall

\title{
Premature coronary artery disease shows no evidence of linkage to loci encoding for tissue inhibitors of matrix metalloproteinases
}

Received: 30 May 2003/ Accepted: 24 July 2003/Published online: 19 September 2003

(C) The Japan Society of Human Genetics and Springer-Verlag 2003

\begin{abstract}
Tissue inhibitors of metalloproteinases (TIMP1, TIMP2, TIMP3) are naturally occurring inhibitors of matrix metalloproteinases (MMPs). It has been proposed that MMPs have a role in weakening the fibrous cap and subsequent plaque rupture. We hypothesized that TIMP polymorphisms could predispose to premature coronary artery disease. As a first step, we examined the relevant loci using a linkage approach. Sibling pairs recruited for the British Heart Foundation (BHF) Family Heart Study with premature coronary artery disease were examined. Two to three microsatellite markers were examined per TIMP gene. These markers were either intragenic or very close to the locus encoding for the gene. Products were analyzed by capillary gel electrophoresis. Single and multipoint linkage analysis based on the likelihood ratio test was performed using SPLINK and Mapmaker/Sibs software; 417 families were genotyped consisting of 385 sibling pairs, 27 trios, and five sets of four siblings. We were unable to detect linkage of premature coronary artery disease to loci encoding for TIMP1-3. Polymorphisms of the tissue inhibitors of $M M P$ genes do not predispose to premature coronary artery disease in an epidemiologically significant way.
\end{abstract}

Keywords Coronary artery disease $\cdot$ Linkage analysis $\cdot$ Candidate genes $\cdot$ Tissue inhibitors of

M. F. Dorsch · R. A. Lawrance - A. Maqbool - N. P. Durham S. Ellis · S. G. Ball · A. J. Balmforth · A. S. Hall ( () Institute for Cardiovascular Research, University of Leeds, Leeds General Infirmary, G-Floor, Jubilee Wing,

Leeds, LS2 9JT, UK

E-mail: a.s.hall@leeds.ac.uk

Tel.: + 44-113-3926526

Fax: + 44-113-3925405

J. A. Barrett · T. Bishop

Cancer Research UK, Clinical Center in Leeds, Leeds, UK

N. J. Samani

Division of Cardiology, University of Leicester, Leicester, UK metalloproteinases $\cdot$ Arteriosclerosis $\cdot$ Extracellular matrix

\section{Introduction}

The stability of an arteriosclerotic plaque depends on the structural integrity of its fibrous cap. Therefore, dissolution of the extracellular matrix by matrix metalloproteinases (MMPs), which are produced by macrophages and smooth muscle cells, is a likely contributing mechanism to plaque rupture (Richardson et al. 1989. Libby, 1995). MMPs 1, 2, 3 and 9 have been discussed in the context of coronary artery disease (CAD) (Newby et al. 1999).

Tissue inhibitors of metalloproteinases (TIMPs) are naturally occurring inhibitors of MMPs. So far, four members of the TIMP family have been described. They are encoded by genes on four different chromosomes (X, 17, 22, 3). TIMP4 was described more recently, is not well characterized compared with the others, and has therefore been omitted from this study. TIMP1, TIMP2, and TIMP3 are all secreted by smooth-muscle cells in vitro and have similar inhibitory activity against MMPs (Apte et al. 1995, Galis et al. 1994).

TIMPs reduce MMP activity (Galis et al. 1994). Consequently, increased expression of TIMPs might counteract MMP action on the fibrous cap of an arteriosclerotic plaque and prevent plaque rupture (Kai et al. 1998, Fabunmi et al. 1998). Increased levels of MMPs and in situ matrix-degrading activity in the vulnerable shoulders of human atheroma have been demonstrated. It has been suggested that these were overcoming inhibition by TIMPs making the plaque prone to rupture (Galis et al. 1994, Galis et al. 2002). In support of this hypothesis, analysis of human coronary arteriectomy specimens has revealed uniformly active synthesis of MMP-9 by macrophages and smooth-muscle cells in 
lesions of patients with unstable versus stable angina (Brown et al. 1995).

TIMP1 and TIMP2 secretion was analyzed in cultured aortic explants from normal and cholesterol-fed rabbits. Increased TIMP expression was found in the cholesterol-fed animals (Zaltsman et al. 1999). Fabunmi and colleagues (Fabunmi et al. 1998) demonstrated increased TIMP3 expression and presence of TIMP1 and TIMP2 in plaque shoulders, intimal-medial borders, and areas overlying the lipid core of the human atheroma. A working hypothesis suggested that the increased TIMP expression could at least, in part, counterbalance increased MMP activity and therefore positively influence plaque stability.

Genetic variations of MMPs have been an area of great interest. Their potential associations with coronary artery disease have been described and have recently been reviewed in detail (Henney et al. 2000, Ye 2000). Multiple TIMP gene mutations have been described and have been related to a number of noncardiovascular diseases. Sorsby's fundus dystrophy, a rare dominantly inherited degenerative disease of the retina, has been causally related to mutations in the gene encoding for TIMP3 (Weber et al. 1994, John et al. 1998). In a further small study of 128 patients, TIMP2 polymorphisms have been associated with chronic obstructive lung disease (Hirano et al. 2001). However, to date no study on genetic variation in the TIMP genes and coronary artery disease have been reported. The objective of this study was to seek evidence for involvement of genetic variation at loci for TIMP 1-3 in the etiology of premature coronary heart disease.

We decided to use a candidate gene approach using linkage analysis with microsatellite markers to investigate specific regions using an initial 417 families with premature coronary artery disease. This approach has been successful in the study of a number of disease states including obesity and rheumatoid arthritis (Bray et al. 1999, John et al. 1998). Microsatellite markers are selected that are located either within the genes of interest or close to them.

Compared to the widely used association studies, our linkage candidate approach has the additional advantage that multiple single nucleotide polymorphisms (SNPs) and also unknown SNPs are studied as a group (haplotype). Therefor, if significant linkage is found, further studies can be undertaken to identify precisely the causative polymorphism. However importantly, if no evidence of linkage can be found, all SNPs within the relevant area are excluded as risk-carrying polymorphisms at the calculated level of gene-specific risk or z-score.

\section{Subjects and methods}

\section{Subjects}

Families with at least two affected sibling pairs from the BHF Family Heart Study were used for the purpose of this study. Entry criteria required both siblings to have coronary artery disease at the age of 65 or younger. Coronary artery disease was defined as at least one of the following: acute myocardial infarction, the need for coronary artery bypass grafting or angioplasty (with or without stenting), or typical angina supported by a positive exercise test or angiogram showing significant coronary atheroma (at least one lesion greater than $50 \%$ ). All cases were validated by reference to their medical records.

The project was performed in accordance with the principles stated in the Declaration of Helsinki. The final protocol, the patient information sheet, and the consent form were approved by multicenter (MREC) and local (LREC) research ethics committees.

\section{Selection of microsatellite markers}

These were identified from a combination of internet sites - $\mathrm{http}$ :// www.gdb.org, http://www.genlink.wustl and http:// www.ncbi.nlm.nih.gov.genemap98. Selection criteria included proximity to the gene of interest, maximal heterozygosity, and PCR-product size range. We restricted our search to well-documented markers with proven annealing specificity such as those on the Genethon and Marshfield map. Two or three markers per gene of interest were chosen to increase power. Commercial markers were used (PE Applied Biosystems, Kelvin Close, Cheshire, UK; MWG AG Biotech, Anzinger Strasse 7a, Ebersberg, Germany). Each forward primer had one of three fluorescent phosphoramidite dyes attached at the 5' end, i.e., FAM (carboxyfluorescin), HEX (4, 7, 2', 4', 5', 7'-Hexachloro-6carboxyfluorescin or NED (4, 7, 2', 7'Tetrachloro-6-carboxyfluorescin) to enable overlapping PCR to be distinguished by dye color. Table 1 gives important information about the selected markers. Fig. 1 displays the position of the markers in relation to the gene of interest on the physical map.

\section{Laboratory-based analysis}

DNA was extracted using the Puregene kit (Gentra Systems, Minneapolis, USA). Conventional PCR methods were used to amplify the genomic area of interest and $10 \mu \mathrm{l}$ reactions with $25 \mathrm{ng}$ of human genomic DNA were used. All other PCR components, i.e., PCR buffer, magnesium chloride, the four deoxynucleotide triphosphates (dNTPs), and a DNA polymerase enzyme were purchased from a commercial vendor (PE Applied Biosystems, Kelvin Close, Cheshire, UK).

PCR was performed using a thermal cycler (Gene Amp PCR system 9600 and Gene Amp PCR system 9700, PE Applied Biosystems, Kelvin Close, Cheshire, UK). Initially, the PCR product was denatured for $15 \mathrm{~min}$ at $95^{\circ} \mathrm{C}$. Then 35 cycles of the following stages were repeated: denaturing at $95^{\circ} \mathrm{C}$ for $30 \mathrm{~s}$ followed by annealing at the optimized temperature (see Table 1) and a $45 \mathrm{~s}$ extension at $72^{\circ} \mathrm{C}$. After a final extension time of $72^{\circ} \mathrm{C}$ for $10 \mathrm{~min}$, the samples were cooled to $4^{\circ} \mathrm{C}$ and kept at this temperature until they were pooled for the sequencing process.

\section{Genotyping}

An ABI PRISM 3700 DNA Analyzer (PE Applied Biosystems) was used for genotyping. A loading solution of fragment size standards (0.06 $\mu \mathrm{l}$; Genescan 400 ROX Size, Applied Biosystems) with $10 \mu \mathrm{l}$ of Hi-Di formamide (Applied Biosystems) and $1 \mu \mathrm{l}$ of pooled PCR product was prepared. Samples were prepared in 96-well plates (MicroAmp Optical 96-Well Reaction Plate, Applied Biosystems) and automatically loaded onto the loading bar in the electrophoresis chamber. PCR fragments were separated using 3700 POP6 polymer with a running temperature of $50{ }^{\circ} \mathrm{C}$ at 7,500 volts for $1.4 \mathrm{~h}$.

Allele assignment was undertaken using GeneMapper 1.0.2 (PE Applied Biosystems, Kelvin Close, Cheshire, UK). This is a software package designed to provide automated genotyping for high throughput genetic linkage analysis. All called genotypes were 

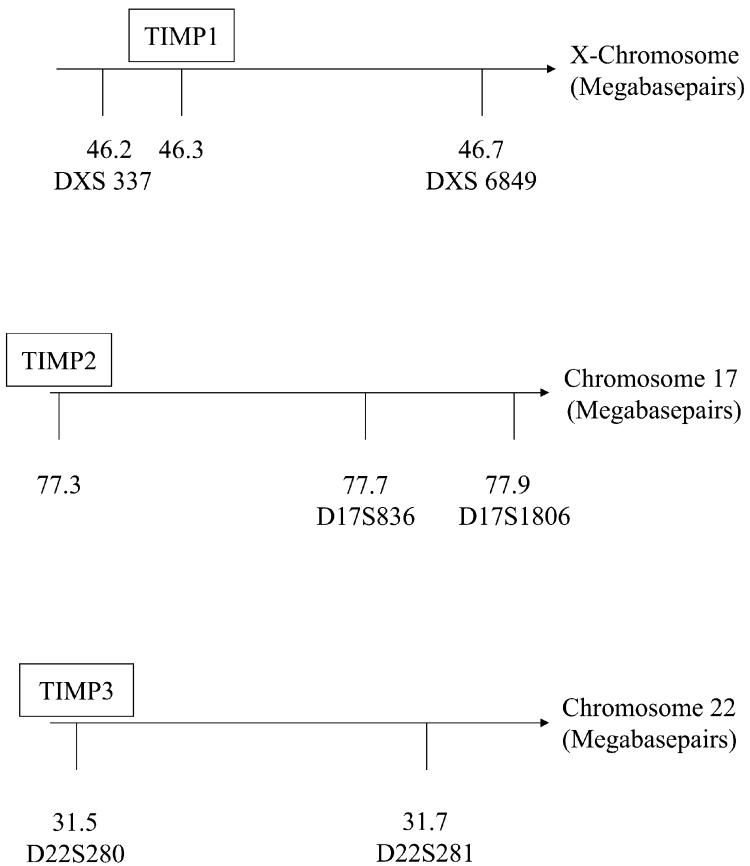

Fig. 1 Position of the markers used in relation to the gene of interest (physical position of marker D22S275 unknown, maximal genetic distance 3.4 centiMorgan from TIMP3). Note different scale for TIMP3

manually double-checked, and the respective PCR reaction was repeated when the operator did not agree with the automated allele calling. A subset of alleles was double-checked independently by a second operator. Interobserver agreement was greater than $98 \%$. Two samples from the initial 96-well plate were used as controls on subsequent runs to ensure consistency between runs.

Data analysis

PDF-output files produced by GeneMapper were read into the software program Genetic Analysis Software (GAS) (Alan Young, Oxford University, UK) that has two functions: It checks the genetic data for compliance with the principles of Mendelian inheritance and merges the two input files (genotyping file and file with information on families) into an output file recognized by the downstream programs SPLINK (David Clayton, Cambridge Institute, UK) and Mapmaker/Sibs (Center for Genome Research, Massachusetts Institute of Technology, Cambridge, MA, USA).

SPLINK, a program for single-point linkage analysis of affected sibling pairs uses maximum likelihood analysis to estimate the probability that two affected siblings share 0,1 , and 2 autosomal marker haplotypes identically by descent (IBD). For $\mathrm{X}$-chromosomal markers, the maternal haplotype only is considered. Where parental data are absent, the IBD sharing is estimated by considering all possible parental haplotypes weighted according to allele frequencies, which are estimated from the data. Trios or larger groups of siblings were analyzed by considering all pair-wise comparisons and weighting by two thirds for trios and one half for quartets. The likelihood was constrained to a solution within the "possible triangle" constraints corresponding to plausible genetic models (Holmans 1993). Version 1.04 was used and run on a MS DOS platform.

Repeated single-point linkage analysis for markers at a given locus does not exploit the full inheritance information available, and considerable additional power can be gained by the simultaneous study of multiple markers, particularly in the absence of parental DNA. Therefore, multipoint analysis was performed using 
the UNIX-based software program Mapmaker/Sibs version 2.0. This employs a rapid algorithm for multipoint likelihood computation to completely characterize the probability distribution of sharing 0,1 , or 2 haplotypes for every sib pair at every point in the genome (Kruglyak and Lander 1995). Possible triangle constraints were still used, and the same weighting scheme was applied for larger sibships. The program implemented a method for X-chromosomal markers that considers brother-brother, brother-sister, and sister-sister pairs separately and then combines the results (Cordell et al. 1995).

Utilizing Mapmaker/Sibs, we also performed exclusion mapping to determine a gene-specific sibling-relative risk for premature coronary artery disease that could be excluded for each locus. An LOD score of -2 was taken to represent a 100:1 likelihood ratio against a locus with this effect in the region. For an X-linked disease, the sibling-relative risk can differ depending on sibling gender, so results were expressed in terms of the degree of IBD sharing between sibling pairs that can be excluded.

The described process of data analysis was repeated for prespecified subgroups, i.e., patients with stable versus unstable coronary artery disease and a subgroup where we considered male sib-pairs only.

\section{Power considerations}

Individual loci affecting a complex trait may have small effects and can be difficult to detect. Therefore, the size of the study is critical in assessing results. A priori power calculations are difficult because relative risk, allele frequency, and mode of inheritance are unknown, but some examples are given below.

Assuming zero recombination, fully informative markers and a significance level of $0.05,400$ sibling pairs give over $80 \%$ power to detect a relative risk of 2.5 to heterozygotes carrying an allele with frequency 0.3 assuming a multiplicative model. This is equivalent to a locus-specific sibling risk ratio of 1.24 . The required sample size rises to 423 for an allele frequency of 0.2 . For a dominant model, $410 \mathrm{sib}$ pairs give $80 \%$ power if the allele frequency is 0.2 , and carriers of the allele are at four times the risk of noncarriers (sibling risk ratio 1.23 ).

\section{Results}

The population for this study were siblings from 417 families (871 individuals) containing at least two siblings affected with premature coronary artery disease. A total of 385 pairs, 27 trios, and five quartets were analyzed. This corresponds to 496 sibling pair comparisons. Using the usual weighting scheme of two thirds for trios and one half for quartets resulted in 454 weighted sib pair comparisons. Baseline characteristics for the whole cohort and the prespecified subgroups are given in Table 2 .

The results of the multipoint analysis are summarized in Tables 3 and 4. No significant linkage to any of the loci examined was demonstrated. Single-point analysis reflected the multipoint analysis (results not shown).

Using the exclusion mapping function of Mapmaker/ Sibs and the exclusion criterion of an LOD score less than -2 , we were able to rule out a gene-specific sibling relative risk of 1.26 and 1.54 for TIMP2 and TIMP3 respectively. For TIMP1 on the $\mathrm{X}$-chromosome we were able to

Table 2 Baseline characteristics for study participants. $M I$ myocardial infarction, $C A D$ coronary artery disease

\begin{tabular}{|c|c|c|c|c|}
\hline & Whole cohort & Male sib pairs & Pairs with MI & Pairs with stable CAD \\
\hline Families & 417 & 244 & 175 & 66 \\
\hline Weighted sib-pair comparisons ${ }^{a}$ & 496 & 275 & 198 & 76 \\
\hline Gender ( $\%$ female $)$ & 26 & 0 & 21 & 40 \\
\hline Mean age (SD) at first cardiac event (years) & $52.5(8)$ & $51.8(8)$ & $51.8(8)$ & $53.3(8)$ \\
\hline Mean age (SD) at interview (years) & $62(7)$ & $61(7)$ & $62(8)$ & $60(7)$ \\
\hline Body mass index (kilogram $/ \mathrm{meter}^{2}$ ) & $27.9(4.0)$ & $27.9(3.4)$ & $27.5(3.7)$ & $29(4.3)$ \\
\hline Myocardial infarction & $62 \%$ & $66 \%$ & $100 \%$ & $0 \%$ \\
\hline Coronary artery bypass grafting & $50 \%$ & $54 \%$ & $41 \%$ & $61 \%$ \\
\hline Percutaneous coronary intervention & $22 \%$ & $21 \%$ & $16 \%$ & $28 \%$ \\
\hline Angina & $76 \%$ & $76 \%$ & $63 \%$ & $98 \%$ \\
\hline Hypertension & $41 \%$ & $38 \%$ & $34 \%$ & $48 \%$ \\
\hline Hypercholesterolemia & $76 \%$ & $75 \%$ & $75 \%$ & $83 \%$ \\
\hline Diabetes & $11 \%$ & $11 \%$ & $10 \%$ & $8 \%$ \\
\hline Current or previous smokers & $75 \%$ & $78 \%$ & $77 \%$ & $67 \%$ \\
\hline
\end{tabular}

${ }^{\mathrm{a} A s}$ sib-pair comparisons are not independent for families with more than 2 siblings, these are weighted ( $2 / 3$ for trios, $1 / 2$ for quartets)

Table 3 Autosomal candidates: LOD scores and estimates for IBD sharing for 0,1 and 2 alleles for the whole group and prespecified subgroups on multipoint linkage analysis. $n$ number of individuals, $M I$ myocardial infarction, $I B D$ identical by descent, $C A D$ coronary artery disease

\begin{tabular}{llllll}
\hline Gene & Marker & $\begin{array}{l}\text { Whole cohort }(n=871) \\
\text { LOD score (IBD sharing) }\end{array}$ & $\begin{array}{l}\text { Male sib pairs }(n=503) \\
\text { LOD score (IBD sharing) }\end{array}$ & $\begin{array}{l}\text { MI sib pairs }(n=361) \\
\text { LOD score (IBD sharing) }\end{array}$ & $\begin{array}{l}\text { Stable CAD dis. pairs }(n=137) \\
\text { LOD score }(I B D ~ s h a r i n g)\end{array}$ \\
\hline TIMP2 & D17S1806 & $0.000(0.25 / 0.5 / 0.25)$ & $0.000(0.25 / 0.5 / 0.25)$ & $0.000(0.25 / 0.5 / 0.25)$ & $0.000(0.25 / 0.5 / 0.25)$ \\
& D17S836 & $0.000(0.25 / 0.5 / 0.25)$ & $0.000(0.25 / 0.5 / 0.25)$ & $0.000(0.25 / 0.5 / 0.25)$ & $0.000(0.25 / 0.5 / 0.25)$ \\
TIMP3 & D22S275 & $0.459(0120.22 / 0.5 / 0.28)$ & $0.239(0.22 / 0.5 / 0.28)$ & $0.049(0.24 / 0.5 / 0.26)$ & $0.696(0.17 / 0.5 / 0.33)$ \\
& D22S280 & $0.440(0.22 / 0.5 / 0.28)$ & $0.182(0.23 / 0.5 / 0.27)$ & $0.018(0.24 / 0.5 / 0.26)$ & $0.733(0.17 / 0.5 / 0.33)$ \\
& D22S281 & $0.418(0.22 / 0.5 / 0.28)$ & $0.186(0.23 / 0.5 / 0.27)$ & $0.031(0.24 / 0.5 / 0.26)$ & $0.737(0.16 / 0.5 / 0.34)$ \\
\hline
\end{tabular}


Table 4 X-chromosomal candidate (TIMP1): LOD scores and estimates for identical by descent (IBD) sharing for 0 and 1 maternal alleles for the whole group and prespecified subgroups on multipoint linkage analysis. $n$ number of individuals, $M I$ myocardial infarction, $C A D$ coronary artery disease

\begin{tabular}{|c|c|c|c|c|c|c|c|c|}
\hline \multirow[t]{2}{*}{ Gene } & \multirow[t]{2}{*}{ Marker } & \multirow[t]{2}{*}{ Pairs } & \multicolumn{2}{|c|}{ Whole cohort $(n=871)$} & \multicolumn{2}{|c|}{ MI sib pairs $(n=361)$} & \multicolumn{2}{|c|}{$\begin{array}{l}\text { Stable CAD dis. pairs } \\
(n=137)\end{array}$} \\
\hline & & & LOD score ${ }^{\mathrm{a}}$ & IBD sharing & LOD score ${ }^{a}$ & IBD sharing & LOD score ${ }^{a}$ & IBD sharing \\
\hline \multirow[t]{4}{*}{ TIMP1 } & \multirow[t]{3}{*}{ DXS 337} & Brother-brother & 0.000 & $0.50 / 0.50$ & \multirow[t]{3}{*}{0.000} & $0.50 / 0.50$ & \multirow[t]{3}{*}{0.189} & $0.50 / 0.50$ \\
\hline & & Brother-sister & & $0.50 / 0.50$ & & $0.50 / 0.50$ & & $0.39 / 0.61$ \\
\hline & & Sister-sister & & $0.50 / 0.50$ & & $0.50 / 0.50$ & & $0.50 / 0.50$ \\
\hline & DXS 6849 & Brother-brother & 0.000 & $0.50 / 0.50$ & 0.000 & $0.50 / 0.50$ & 0.208 & $0.50 / 0.50$ \\
\hline
\end{tabular}

${ }^{a}$ LOD-score given is the total LOD-score for brother-brother, brother-sister, and sister-sister pairs

exclude a sharing probability of the maternally inherited allele of 0.56 or more, assuming the same sharing probability applied to brother-brother, brother-sister, and sister-sister pairs (appropriate for a recessive model).

\section{Discussion}

We were unable to detect linkage of premature coronary artery disease to the loci encoding for TIMP1, TIMP2, or TIMP3 in either our whole cohort or prespecified subgroups. For all loci of interest, at least two markers with small recombination fraction and high heterozygosity were used, further enhancing the power of the analysis.

There are different potential explanations why a linkage study for a candidate gene may be negative. First and foremost, the candidate investigated might not have a polymorphism that increases susceptibility to premature coronary artery disease. Although a potential pathophysiological basis for the involvement of the TIMP genes in premature coronary artery disease has been outlined, it is important to acknowledge that despite recent advances, the exact pathophysiology of arteriosclerosis is still incompletely understood. Furthermore, even if one of the candidates discussed had a functional gene polymorphism leading to a defective protein, it might not clinically manifest itself, as another gene product might compensate. A possible explanation may be that all TIMPs have broadly similar inhibitory action of all MMPs, and so there seems to be little substrate specificity (Apte et al. 1995). This could function as a biological "backup mechanism" so that even if there were a mutation that would lead to an abnormal TIMP gene product, this might have little pathophysiological consequence because the function of the protein (inhibition of matrix metalloproteinases) could be taken over by one of the other functional TIMPs.

Alternatively, it is possible that the clinically relevant mutations are confined to TIMP4, the remaining member of the TIMP family, which has not been analyzed as a candidate gene in this study.

Furthermore, conclusions drawn are valid only for the population investigated. It is conceivable that the genes, for which no linkage to premature coronary artery disease was found in the UK population, might still have important functional polymorphism in a cohort of patients from a different population.

Finally, we might not have been able to detect linkage because of insufficient power of our study. This could be a particular problem in the analyzed subgroups, for example TIMP3 in siblings with stable CAD. Although the maximal LOD score of 0.74 in this group was not of statistical significance, it is possible that a small effect might be demonstrable if we would have had a larger sample size. Additionally, if there was a lot of genetic heterogeneity, i.e., different genes being responsible for different so far indistinguishable types of CAD, the power calculations might be misleading and the gene(s) could have an important effect in a so-far unrecognized subgroup of all cases.

However, it is important to note that the number of affected sibling pairs recruited into our study was larger than that of all four recently published genome-wide screens (Pajukanta et al. 2000, Francke et al. 2001, Broeckel et al. 2000, Harrap et al. 2002). Compared to genome-wide screens, our study will also have superior power to exclude linkage to the relevant loci because the recombination fraction approaches zero in our design (Risch 1990a, John et al. 1997). The sensitivity of our method is further increased by using more than one marker per candidate locus to compensate for incompletely informative markers (Risch 1990a, Risch 1990b). Therefore, we are close to the assumptions of zero recombination and full informativeness of the markers, which are generally assumed in power calculations.

We were able to exclude a gene-specific relative sibling risk of 1.26 and 1.54 for TIMP2 and TIMP3 respectively. For the X-chromosomal TIMP1, we excluded linkage for allele sharing with a z-score of 0.56 or greater. This level of exclusion was sufficient to rule out a clinically relevant effect, i.e., an effect that would have an impact on predicting prognosis in a given patient or an effect that would be worth targeting with specifically designed drugs. It is therefore conceivable that one or more of our candidates, without detectable linkage to premature coronary artery disease, still has a rare polymorphism or a polymorphism with a small 
genotypic risk ratio that predisposes the carrier to premature coronary artery disease. However, both of these scenarios would have less impact on public health issues or the development of new treatment strategies, although particularly a rare polymorphism with a strong effect could be of great importance to the individual.

Our findings are also supported by the results of the genome-wide screens that have been published in the field of premature coronary artery disease (Pajukanta et al. 2000, Francke et al. 2001, Broeckel et al. 2000, Harrap et al. 2002). In none of these studies have potentially important LOD scores $(>1)$ been reported for these regions.

In summary, we were unable to detect linkage of premature coronary artery disease to the loci encoding for TIMP1, TIMP2, or TIMP3. This makes it unlikely that a polymorphic variant of one of the respective genes predisposes to premature coronary artery disease.

\section{References}

Apte S, Olsen B, Murphy G (1995) The gene structure of tissue inhibitor of metalloproteinases (TIMP3) and its inhibitory activities define the distinct TIMP gene family. J Biol Chem 270:14313-14318

Bray MS, Boerwinkle E, Hanis CL (1999) Linkage analysis of candidate obesity genes among the Mexican-American population of Starr County, Texas. Genet Epidemiol 16(4):397-411

Broeckel U, Hengstenberg C, Mayer B, et al (2000) A comprehensive linkage analysis for myocardial infarction and its related risk factors. Nature Genetics 30:210-214

Brown DL, Hibbs MS, Kearney M, et al (1995) Identification of 92-kD gelatinase in human coronary atherosclerotic lesions: association of active enzyme synthesis with unstable angina. Circulation 91:2125-31

Cordell HJ, Kawaguchi Y, Todd JA, et al (1995) An extension of the maximum LOD score method to X-linked loci. Ann Hum Genet 59:435-449

Fabunmi RP, Sukhova GK, Sugiyama S, et al (1998) Expression of tissue inhibitor of metalloproteinases --3 in human atheroma and regulation in lesion-associated cells: a potential protective mechanism in plaque stability. Circ Res 83:270-278

Francke S, Manraj M, Lacquemant C, et al (2001) A genome wide scan for coronary heart disease suggests in Indo-Mauritians a susceptibility locus on chromosome $16 \mathrm{p} 13$ and replicates linkage with the metabolic syndrome on 3q27. Hum Mol Genet 24(10) 2751-2765

Galis ZS, Khatri JJ (2002) Matrix metalloproteinases in vascular remodelling and atherogenesis. The good, the bad, and the ugly. Circ Res 90 251-262

Galis ZS, Muszynski M, Sukhova GK (1994) Cytokine-stimulated human vascular smooth muscle cells synthesize a complement of extracellular enzymes required for extracellular matrix degradation. Circ Res 75:181-189
Harrap SB, Zammit KS, Wong ZY, et al (2002) Genome-wide linkage analysis of the acute coronary syndromes suggest a locus on chromosome 2. Arterioscler Thromb Vasc Biol 22(5):874-878

Henney AM, Ye S, Zhang B, et al (2000) Genetic diversity in the matrix metalloproteinase family: Effects on function and disease progression. Annals of the New York Academy of Science 902:27-38

Hirano K, Sakamoto T, Uchida Y, et al (2001) Tissue inhibitor of metalloproteinases-2 geen polymorphisms in chronic obstructive pulmonary disease. Eur Respir J 18(5):741-743

Holmans (1993) Asymptomatic properties of affected-sib-pair linkage analysis. Am J Hum Genet 52:362-374

John S, Hajeer A, Marlow A, et al (1997) Investigation of candidate susceptibility genes in rheumatoid arthritis: Principles and strategies. J Rheumatol 24:199-201

John S, Korman Y, Ollier W, et al (1998) Integrated microsatellites markers suitable for genetic studies of cytokine genes. Cytokine $10: 466-470$

Kai H, Ikeda H, Yasukawa H, et al (1998) Peripheral blood levels of matrix metalloproteases- 2 and -9 are elevated in patients with acute coronary Syndromes. J Am Coll Cardiol 32:368-372

Kruglyak L, Lander ES (1995) Complete multipoint sib-pair analysis of qualitative and quantitative traits. Am J Hum Genet 57:439-454

Langton KP, McKie N, Curtis A, et al (2000) A novel tissue inhibitor of metalloproteinas-3 mutation reveals a common molecular phenotype in Sorsby's fundus dystrophy. J Biol Chem 275:27027-27031

Libby P (1995) Molecular basis of acute coronary syndromes. Circulation 91:2844-2850

Newby AC, Zaltsman AB (1999) Fibrous cap formation or destruction- the critical importance of vascular smooth muscle cell proliferation, migration and matrix formation. Cardiovasc Res 41:345-360

Pajukanta P, Cargill M, Viitanen L, et al (2000) Two loci on chromosomes 2 and $\mathrm{X}$ for premature coronary heart disease identified in early- and late-settlement populations of Finland. Am J Hum Genet 67:1481-1493

Richardson P, Davies M, Born G (1989) Influence of plaque configuration and stress distribution on fissuring of coronary arteriosclerotic plaques. Lancet: 941-944

Risch N (1990a) Linkage strategies for genetically complex traits. II. The power of affected relative pairs. Am J Hum Gen 46: 229-241

Risch N (1990b) Linkage strategies for genetically complex traits. III. The effect of marker polymorphism on analysis of affected relative pairs. Am J Hum Gen 46:242-253

Weber BH, Vogt G, Pruett RC, et al (1994) Mutations in the tissue inhibitor of metalloproteinases-3 (TIMP3) in patients with Sorsby's fundus dystrophy. Nat Genet 8(4):352-356

Ye S (2000) Polymorphism in matrix metalloproteinase gene promoters: implication in regulation of gene expression and susceptibility of various diseases. Matrix Biol 19(7): 623-629

Zaltsman AB, George SJ, Newby AC (1999) Increased secretion of tissue inhibitors of metalloproteinases 1 and 2 from the aortas of cholesterol fed rabbits partially counterbalances increased metalloproteinase activity. Arterioscler Thromb Vasc Biol 19(7):1700-1707 